\title{
Inclusive Deep Inelastic Scattering at HERA
}

\author{
Paul Newman \\ School of Physics \& Astronomy, University of Birmingham, B15 2TT, UK
}

\begin{abstract}
Recent inclusive charged and neutral current scattering data from HERA are presented. Emphasis is placed on the resulting constraints on the proton parton densities and on the influence of low $x$ proton structure on diffraction.
\end{abstract}

Keywords: DIS, PDF, QCD, proton structure, HERA, H1, ZEUS

PACS: PACS 11, 12, 13, 14

\section{INTRODUCTION}

By the completion of its programme in 2007, the HERA collider had delivered an integrated luminosity of around $500 \mathrm{pb}^{-1}$ to each of the H1 and ZEUS experiments. The bulk of the data were taken at a proton beam energy of $E_{p}=920 \mathrm{GeV}$, corresponding to an electron-proton centre-of-mass energy in excess of $300 \mathrm{GeV}$. Small samples at $E_{p}=575 \mathrm{GeV}$ and $E_{p}=460 \mathrm{GeV}$ were also obtained at the end of the running. For much of the data, the lepton beams were longitudinally polarised, with typical polarisations of $30-40 \%$. Sizeable data samples were obtained for both positive and negative lepton helicities with both electron and positron beams.

Among the many physics results obtained [1], those on the inclusive neutral (NC, $e p \rightarrow e X$ ) and charged (CC, $e p \rightarrow v X$ ) current deep inelastic scattering (DIS) cross sections provide the best constraints on the parton densities of the proton and are discussed here [2]. Recently, several combined H1 and ZEUS results have been obtained for these cross sections. The improvement in precision achieved goes beyond the purely statistical, due to the possibility essentially to cross-calibrate between the two experiments and thus reduce the rather different dominating systematics [3]. Using the data from HERA-I (the first running period ending in 2000), a combined set of parton densities (HERAPDF 1.0) has been obtained [4] and is used in most cases for the comparison of standard model predictions with the data here. There is still considerable scope for improvement as these procedures are extended to HERA-II data.

The parton densities at low $x$ are heavily dominated by the gluon density, which is intimately connected with the large rate of diffractive scattering in DIS [5]. Strong interaction dynamics in the presence of a very large parton density remains a rather poorly understood subject, which is being investigated using further recent inclusive HERA data. 


\section{OVERVIEW OF THE DATA}

The kinematics of inclusive DIS are usually described by the variables $Q^{2}$, the modulus of the squared four-momentum transfer carried by the exchanged electroweak gauge boson, and $x$, the fraction of the proton's longitudinal momentum carried by the quark to which the boson couples. The NC process takes place via the exchange of virtual photon and $Z^{0}$ propagators. The cross section can be expressed in the form

$$
\frac{\mathrm{d} \sigma^{\mathrm{NC}}}{\mathrm{d} x \mathrm{~d} Q^{2}}=2 \pi \alpha_{\mathrm{em}}^{2} \cdot\left(\frac{1}{Q^{2}}\right)^{2} \cdot \frac{Y_{+}}{x} \cdot \tilde{\sigma}_{\mathrm{NC}},
$$

where the term $\alpha_{\mathrm{em}}^{2}$ expresses the dominance of photon exchange over most of the phase space, $1 / Q^{4}$ is the photon propagator and the reduced cross section $\tilde{\sigma}_{\mathrm{NC}}$ contains helicity factors, weak terms due to $Z^{0}$ exchange and structure functions related to the parton densities of the proton. The variables $Y_{ \pm}=1 \pm(1-y)^{2}$, dependent on the inelasticity, $y$, express the helicity dependence of the electroweak interaction.

The CC process is purely due to weak interactions. The cross section can be expressed as

$$
\frac{\mathrm{d} \sigma^{\mathrm{CC}}}{\mathrm{d} x \mathrm{~d} Q^{2}}=\frac{G_{F}^{2} M_{W}^{4}}{2 \pi} \cdot\left(\frac{1}{Q^{2}+M_{W}^{2}}\right)^{2} \cdot \frac{1}{x} \cdot \tilde{\sigma}_{\mathrm{CC}},
$$

where the coupling and propagator terms are appropriate to $W$ boson exchange and the reduced cross section term $\tilde{\sigma}_{\mathrm{CC}}$ contains the helicity factors and structure functions.
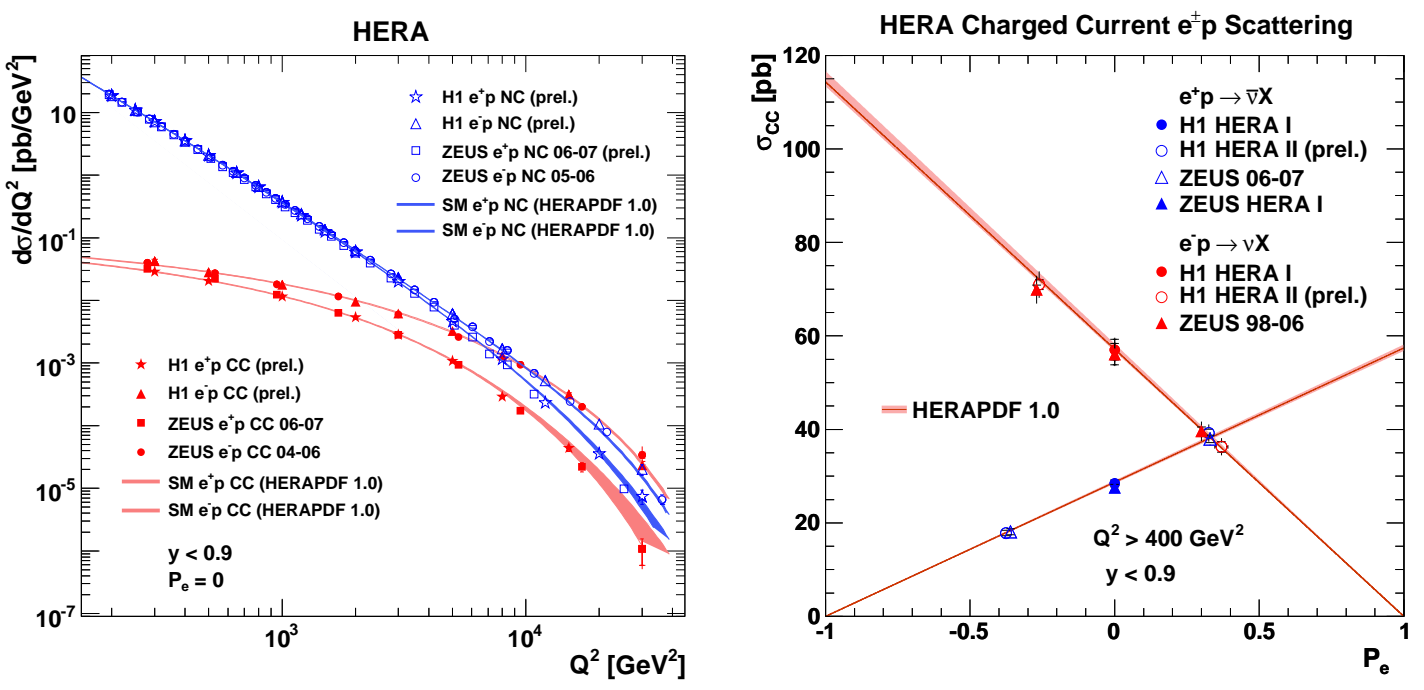

FIGURE 1. (a) Single differential cross sections for charged and neutral current $e^{ \pm} p$ scattering from $\mathrm{H} 1$ and ZEUS. (b) Dependence of the total charged current cross section for $y<0.9$ and $Q^{2}>400 \mathrm{GeV}^{2}$ on the lepton polarisation for $e^{ \pm} p$ scattering, as measured by H1 and ZEUS. In both figures, the data are compared with predictions obtained from the HERAPDF 1.0 parton densities.

Figure 1a shows the single differential cross sections measured using HERA-II data by $\mathrm{H} 1[6,7]$ and ZEUS $[8,9,10,11]$ for charged and neutral current $e^{ \pm} p$ scattering with 
$Q^{2}>200 \mathrm{GeV}^{2}$ and $y<0.9$. For $Q^{2} \ll M_{W}^{2}$, the NC cross section dominates heavily due to the differences between the propagator terms in Eqs. (1) and (2). For $Q^{2} \gtrsim M_{W}^{2}$, the cross sections for $\mathrm{NC}$ and $\mathrm{CC}$ processes become comparable, providing an illustration of electroweak unification with space-like gauge bosons. The remaining differences between the NC and CC cross sections in this large $Q^{2}$ region and the differences between the $e^{+} p$ and $e^{-} p$ cross sections can be understood from the structure of the reduced cross sections $\tilde{\sigma}_{\mathrm{NC}}$ and $\tilde{\sigma}_{\mathrm{CC}}$.

Figure $1 \mathrm{~b}$ shows the polarisation dependence of the charged current $e^{ \pm} p$ scattering cross section [7, 10,11], integrated over wide $Q^{2}$ and $y$ ranges. The data are compatible with the linear dependence expected in the Standard Model and with the disappearance of the cross section for the exchange of right-handed $W$ bosons.
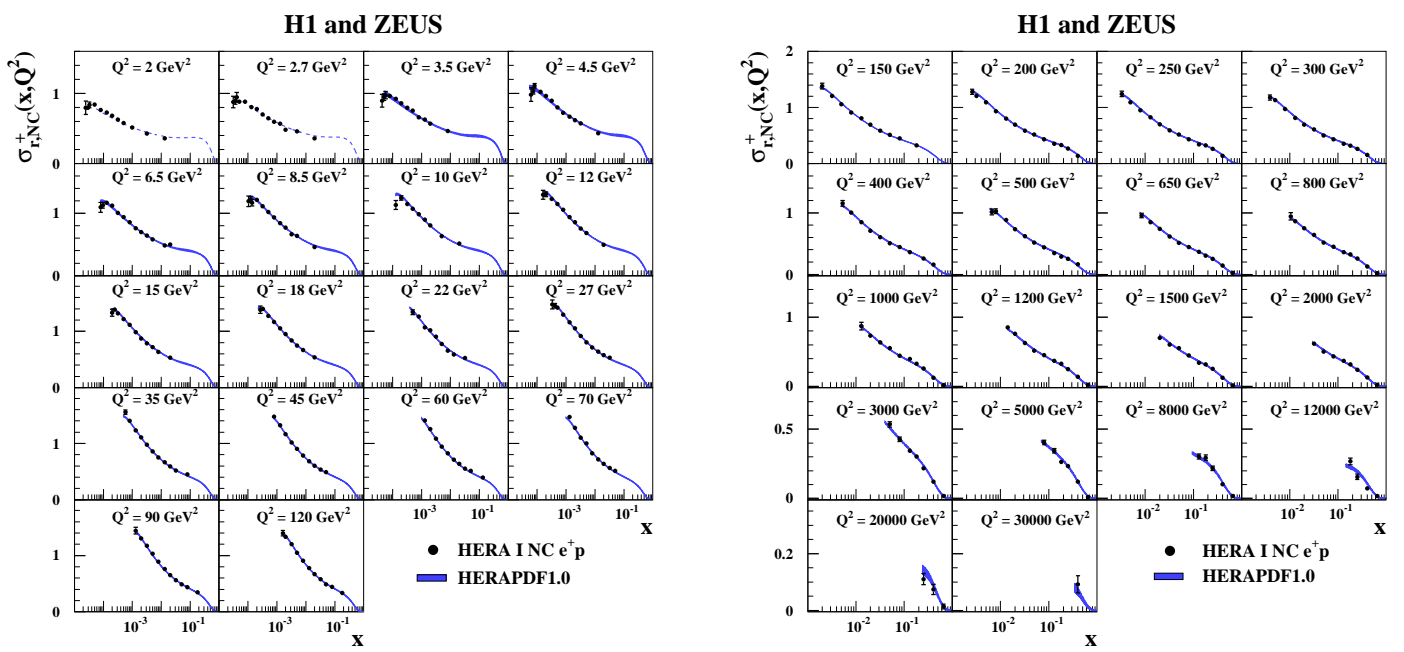

FIGURE 2. The complete set of combined H1 and ZEUS data for the NC reduced cross section.

Combined H1 and ZEUS data from HERA-I [4] are shown in figure 2. 2\% precision is reached across a wide range of the phase space. Further improvements in statistics will improve the high $Q^{2}$ region when the HERA-II measurements are included.

Due to the squared charge coupling $e_{q}$ in the dominant neutral current structure function $F_{2}\left(x, Q^{2}\right)=x \sum_{q} e_{q}^{2}(q+\bar{q})$, the neutral current cross section is most sensitive to the $u$ and $\bar{u}$ quark densities. These NC data also provide the best available constraints on the gluon density via the deviations from Bjorken scaling, caused by gluon radiation. In leading order of QCD, the gluon density can be obtained approximately from $\frac{\partial F_{2}\left(x / 2, Q^{2}\right)}{\partial \ln Q^{2}} \sim \alpha_{S} x g(x)$ [12], such that the strong positive scaling violations at low $x$ are indicative of a large and growing gluon density as $x$ becomes small.

Figure 3 illustrates the sensitivity of the high $Q^{2} \mathrm{NC}$ cross section to electroweak effects. The differences between the $e^{+} p$ and $e^{-} p$ cross sections at large $Q^{2}$ (figure $3 \mathrm{a}$ [4]) arise mainly due to interference between photon and $Z^{0}$ exchange and are encoded in the structure function $x F_{3}^{\gamma Z}=2 x \sum_{q} e_{q} a_{q}(q-\bar{q}) \sim x\left(2 u_{v}+d_{v}\right) / 3$, where $a_{q}$ is the axial coupling of the $Z$ to the quarks. Since $x F_{3}$ measures the difference between the quark and antiquark densities, it is model independently sensitive to the non-singlet valence 

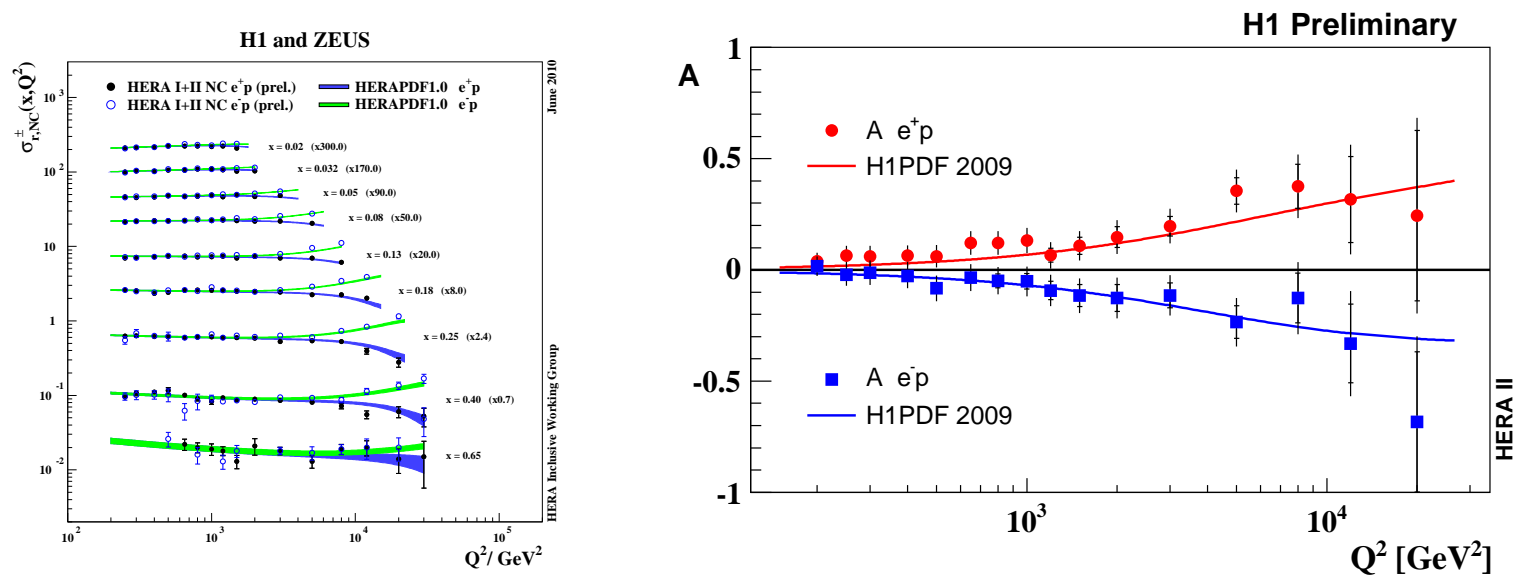

FIGURE 3. (a) Comparison between the $e^{+} p$ and $e^{-} p$ reduced cross sections at large $Q^{2}$. The data are further compared with predictions based on the HERAPDF 1.0 parton densities. (b) Measurements of the asymmetry between positive and negative lepton helicity cross sections for $e^{+} p$ and $e^{-} p$ scattering. The data are compared with an earlier PDF set from H1 [13].

quark densities.

The neutral current polarisation asymmetry (figure $3 \mathrm{~b}[6]$ ) is now measured to be significantly non-zero. This provides complementary information on the valence quark densities via $A \propto\left(1+d_{v} / u_{v}\right) /\left(4+d_{v} / u_{v}\right)$.

Charged current data can be used to constrain the flavour decomposition of the quarks at relatively large $x$, via the charge-sensitive couplings to the $W$ boson. For example, via $\tilde{\sigma}_{\mathrm{CC}}^{+}=x(\bar{u}+\bar{c})+(1-y)^{2} x(d+s)$, the $e^{+} p$ cross section arises dominantly from valence down quark scattering. Via a similar relationship, the $e^{-} p \mathrm{CC}$ cross section provides a further constraint on the up quark density.

\section{PARTON DENSITIES FROM HERA DATA ALONE}

All HERA-I inclusive data have been subjected to a QCD fit in which parton evolution takes place according to next-to-leading order DGLAP. Uncertainties due to the choice of parton parametrisation at the starting scale for evolution are now evaluated, in addition to the usual propagation of experimental and model-dependence uncertainties. The resulting HERAPDF1.0 parton densities [4] are shown in figure 4 at the starting scale for QCD evolution $\left(Q^{2}=1.9 \mathrm{GeV}^{2}\right)$ and at $Q^{2}=10 \mathrm{GeV}^{2}$. Through this relatively small scale increase, the gluon density evolves from a valence-like shape to become the dominant parton density in the low $x$ region (see below). The largest remaining uncertainties appear as $x \rightarrow 1$. Increased statistics at large $Q^{2}$ and data with reduced $E_{p}$ help considerably in this region, as is already becoming clear from preliminary studies including HERA-II data [14, 15].

The HERAPDF 1.0 partons provide a good model for many hadronic final state observables at HERA, as well as Tevatron data, for example on jet production and electroweak gauge boson production [16]. They also provide competitive predictions 

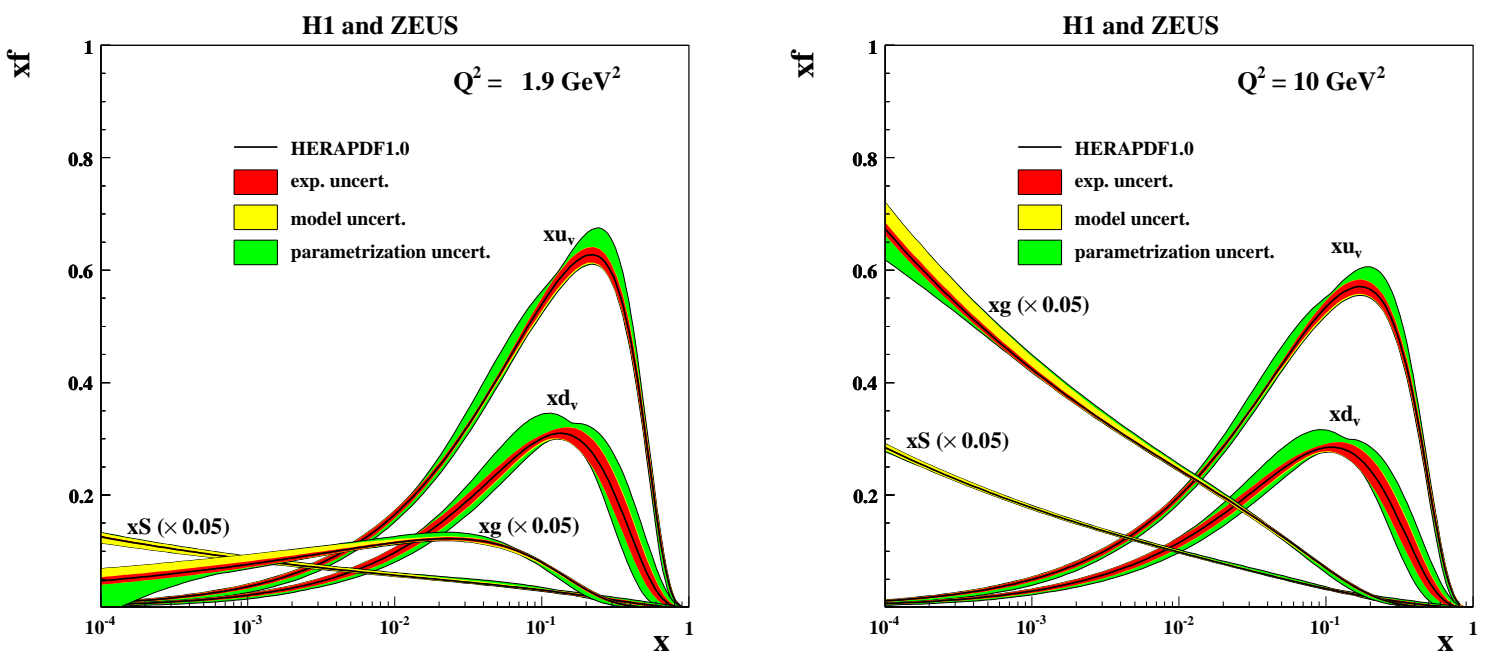

FIGURE 4. HERAPDF 1.0 parton densities at the starting scale for $\mathrm{QCD}$ evolution $Q_{0}^{2}=1.9 \mathrm{GeV}^{2}$ and at $Q^{2}=10 \mathrm{GeV}^{2}$. The gluon and sea quark densities are suppressed by a factor of 20 for visibility.

for LHC cross sections, as illustrated in figure 5. Comparing predictions for example for $W, Z$, Higgs or $t \bar{t}$ cross sections between parton densities obtained with varying procedures and data sets (HERAPDF 1.0, MSTW08 [17], CTEQ6.6 [18] and NNPDF2.0 [19]) suggests that $W$ and $Z$ production cross sections can reliably be predicted to around $5 \%$ precision. The corresponding figures for light Standard Model Higgs and $t \bar{t}$ rates are slightly larger.
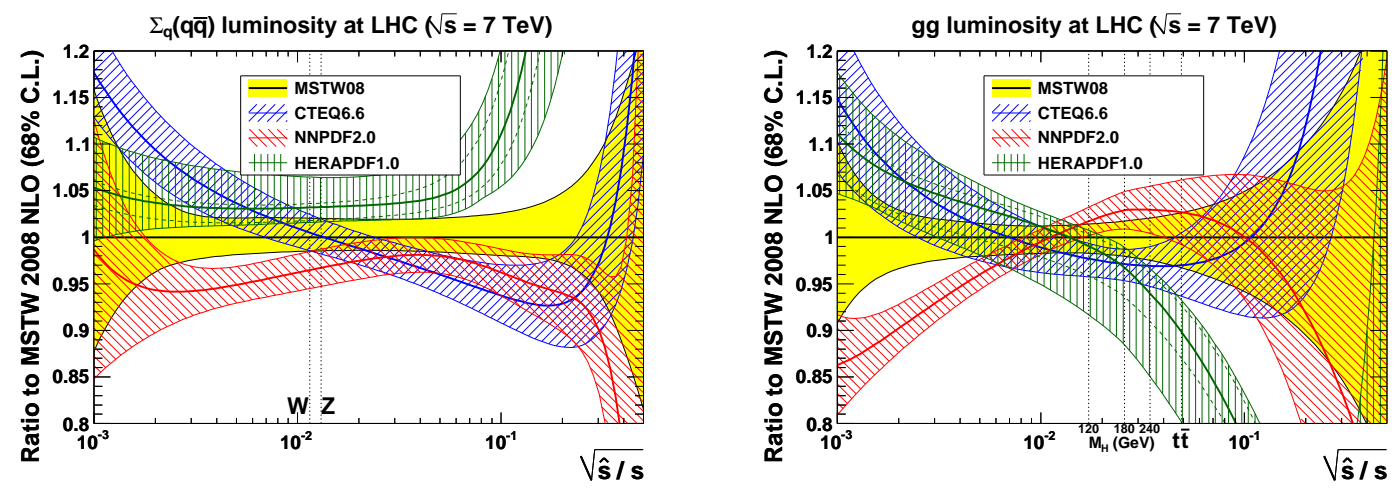

FIGURE 5. Comparison of predictions for LHC parton luminosities between commonly used PDF sets [20]. The band centred around zero indicates the uncertainty in the $q \bar{q}$ or $g g$ luminosity as a function of the product of the $x$ values for the two partons according to MSTW. Other bands indicate the deviations from MSTW of the CTEQ, NNPDF and HERAPDF predictions, as well as the uncertainties intrinsic to each of the fits. 


\section{LOW $x$ ISSUES}

Due to the kinematic restrictions on the $Q^{2}$ range available, the gluon density is poorly constrained for $x \lesssim 10^{-4}$. Together with the large extracted gluon densities in this region, this raises questions about the validity of NLO DGLAP and the possibility of nonlinear contributions to the evolution, as expected for example in colour-glass condensate models [21] and other approaches that contain parton saturation effects [22]. Non-linear gluon dynamics might be observable as a deviation from the observed monotonic rise of the low $x$ NC cross section, which is well parametrised by the form $F_{2}=a\left(Q^{2}\right) x^{-\lambda\left(Q^{2}\right)}$ [23]. The parameter $\lambda$ can be extracted through the logarithmic $x$ derivative at fixed $Q^{2}, \lambda\left(x, Q^{2}\right)=\left(\partial \ln F_{2} / \partial \ln x\right)_{Q^{2}}$. This derivative has been extracted locally from the data based on differences between neighbouring data points. The results [13], shown in figure $6 \mathrm{a}$, are consistent with constant $\lambda$ for $x<10^{-2}$ at each $Q^{2}$ value and thus show no evidence for any taming of the low $x$ rise of the parton densities in the HERA region.
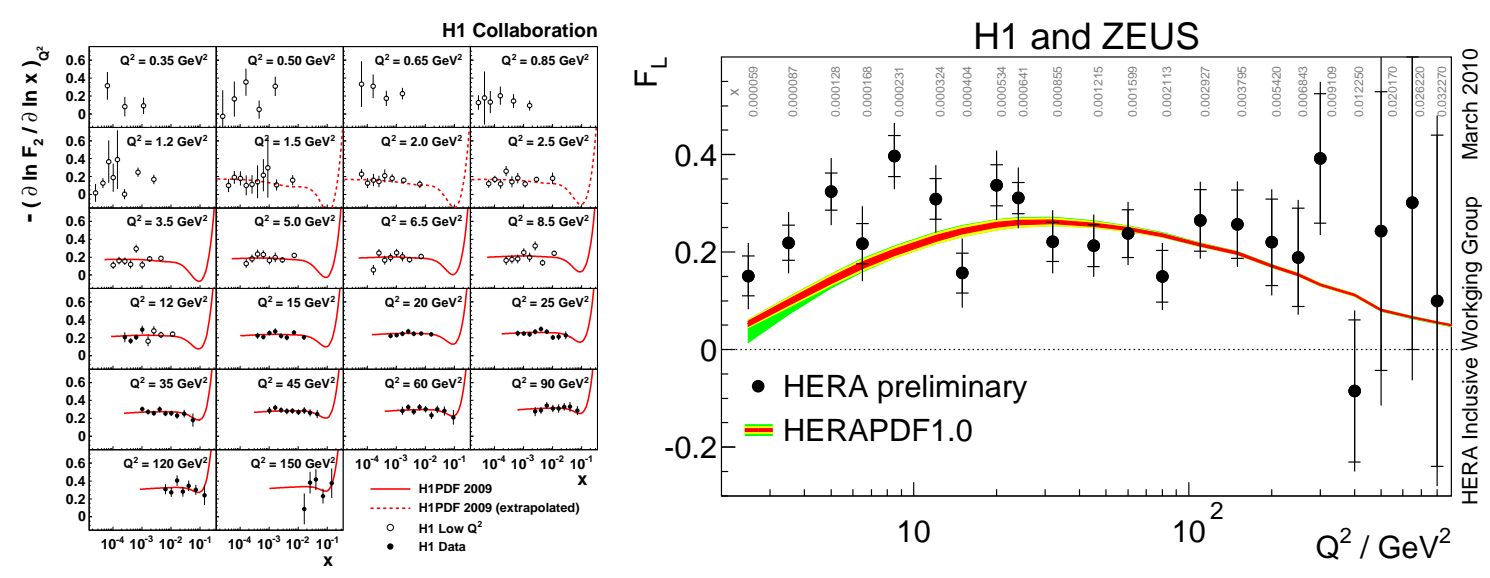

FIGURE 6. (a) Locally extracted values of the logarithmic $x$ derivative of the structure function $F_{2}$, corresponding to the parameter $\lambda$ which describes its low $x$ rise. (b) $Q^{2}$ dependence of the structure function $F_{L}$, as extracted using the $E_{p}$ dependence of the NC DIS cross section.

The issue of the low $x$ gluon density can be investigated directly by measurement of the dependence of the NC cross section on $E_{p}$ at fixed $x$ and $Q^{2}$. This dependence has been used to extract the longitudinal structure function $F_{L}$, which is approximately proportional to the gluon density. Combined $F_{L}$ data from H1 and ZEUS are shown in figure $6 \mathrm{~b}$. The agreement with HERAPDF 1.0 fit predictions is good throughout most of the range, though there are interesting deviations at the lowest $x$ and $Q^{2}$. It remains to be seen whether these deviations are due to new dynamics or whether they reflect the need for higher order DGLAP terms or refined heavy flavour treatment.

\section{SUMMARY}

Combined H1 and ZEUS data from HERA-I provide a new level of precision on the proton quark and gluon densities in the region $10^{-4}<x<10^{-1}$, crucial for future experimentation at the LHC and elsewhere. Further improvements will be made at large 
$x$ when the HERA-II data analysis is completed. There have been considerable recent developments in understanding the region of low $x$ and $Q^{2}$ and testing the range of validity of DGLAP evolution.

\section{ACKNOWLEDGMENTS}

This work was supported in part by a grant from the IPPP, Durham, for which the author is most grateful. Thanks are due to many colleagues who have worked on the H1 and ZEUS experiments for over two decades.

\section{REFERENCES}

1. M. Klein and R. Yoshida, Prog. Part. Nucl. Phys. 61, 343 (2008).

2. Slides at http://www.cs.infn.it/diff2010/talks/Newman1.pdf.

3. H1 Collaboration, Eur. Phys. J. C63, 625 (2009).

4. H1 and ZEUS Collaborations,JHEP 1001, 109 (2010).

5. L. Favart, D. Salek and A. Solano, these proceedings.

6. H1 Collaboration, H1 prelim-09-042.

Available from http: / / www-h1.desy.de/publications/H1preliminary.short_list.html.

7. H1 Collaboration, H1prelim-09-043.

Available from http: // www-h1.desy.de/publications/H1preliminary.short_list.html.

8. ZEUS Collaboration, ZEUS-prel-10-007.

Available from http://www-zeus.desy.de/public_results/publicsearch.html

9. ZEUS Collaboration, Eur. Phys. J. C62, 625 (2009).

10. ZEUS Collaboration, Eur. Phys. J. C61, 923 (2009).

11. ZEUS Collaboration, Eur. Phys. J. C70, 945 (2010).

12. K. Prytz, Phys. Lett. B311, 286 (1993).

13. H1 Colaboration, Eur. Phys. J. C64, 561 (2009).

14. H1 and ZEUS Collaborations, H1 prelim-10-142 and ZEUS-prel-10-018.

Available from http: //www-h1.desy.de/publications/H1preliminary.short_list.html.

15. H1 and ZEUS Collaborations, H1prelim-10-044 and ZEUS-prel-10-008.

Available from http: //www-h1.desy.de/publications/H1preliminary.short_list.html.

16. Plots available at https : / / www. desy.de/h1 zeus/combined_results/benchmark/tev.html.

17. A. Martin et al., Eur. Phys. J. C63, 189 (2009).

18. P. Nadolsky et al., Phys. Rev. D78, 013004 (2008).

19. R. Ball et al., Nucl. Phys. B809, 1 (2008);

R. Ball et al., Nucl. Phys. B823, 195 (2008).

20. G. Watt, PDF Benchmarking for LHC Process, Talk at PDF4LHC Meeting, CERN, March 2010. Available from http: //indico. cern. ch/conferenceDisplay.py? confId=87871.

21. E. Iancu, K. Itakura and S. Munier, Phys. Lett. B590, 199 (2004).

22. K. Golec-Biernat and M. Wüsthoff, Phys. Rev. D59, 014017 (1999).

23. H1 Collaboration, Phys. Lett. B520, 183 (2001). 